\title{
PERFORMANCE OF CAPILLARY DISCHARGE GUIDED LASER PLASMA WAKEFIELD ACCELERATOR*
}

\author{
K. Nakamura ${ }^{\dagger \ddagger}$, E. Esarey, C. G. R. Geddes, A. J. Gonsalves, W. P. Leemans, D. Panasenko \\ C. B. Schroeder, and Cs. Toth, LBNL, Berkeley, California 94720, USA \\ S. M. Hooker, University of Oxford, Oxford OX13PU, UK
}

Abstract

A GeV-class laser-driven plasma-based wakefield accelerator has been realized at the Lawrence Berkeley National Laboratory (LBNL). The device consists of the $40 \mathrm{TW}$ high repetition rate Ti:sapphire LOASIS laser system at LBNL and a gas-filled capillary discharge waveguide developed at Oxford University. The operation of the capillary discharge guided laser plasma wakefield accelerator with a capillary of $225 \mu \mathrm{m}$ diameter and $33 \mathrm{~mm}$ in length was analyzed in detail. The input intensity dependence suggests that excessive self-injection causes increased beam loading leading to broadband lower energy electron beam generation. The trigger versus laser arrival timing dependence suggests that the plasma channel parameters can be tuned to reduce beam divergence.

\section{INTRODUCTION}

The plasma-based accelerator [1] has attracted a lot of attention since it has demonstrated several orders of magnitude larger accelerating gradient compared to conventional radio frequency (RF) accelerators. The concept provides a way to realize a compact accelerator or light source [2]. Furthermore, the electron beams are naturally much shorter in time duration [3] because the characteristic scale length of the accelerating structure is the plasma wavelength, which is typically $\simeq 10 \mu \mathrm{m}$. In case of a laserplasma wakefield accelerator (LWFA), it becomes possible to provide a femto-second particle beam synchronized with a femto-socond laser beam, which may be of benefit to ultra-fast sciences [4].

Recently, a GeV-class laser-driven plasma-based wakefield accelerator has been realized by using a gas-filled capillary discharge waveguide at Lawrence Berkeley National Laboratory (LBNL) $[5,6]$. By guiding a focused highpower laser pulse over a distance 10 times the Rayleigh range, electrons that were self-trapped from the background plasma, were efficiently accelerated up to a $\mathrm{GeV}$ level. The trapping and acceleration processes are complex and are being studied in detail using further experiments and simulation. Here we present a statistical analysis on the operation of a capillary discharge guided laser plasma wakefield accelerator (CDG-LWFA), that gives insight into the underlying physics.

\footnotetext{
- Work supported by DOE grant DE-AC02-05CH11231, DARPA, and and INCITE computational grant.

†Nakamura@lbl.gov

Ialso University of Tokyo, Japan
}

\section{EXPERIMENTAL SETUP}

The schematic of the CDG-LWFA and the diagnostic system is shown in Fig. 1. The laser that was utilized was the high peak power $10 \mathrm{~Hz}$ Ti:sapphire laser system of the LOASIS facility at LBNL [6]. The laser beam was focused onto the entrance of a capillary discharge waveguide by an $1 / 25$ off-axis parabolic mirror. A typical focal spot size was $w_{0} \simeq 25 \mu \mathrm{m}$, which gives a calculated peak intensity $I=2 P / \pi r_{s}^{2} \simeq 4 \times 10^{18} \mathrm{~W} / \mathrm{cm}^{2}$ and a normalized vector potential $a_{0} \simeq 8.6 \times 10^{-10} \lambda[\mu \mathrm{m}] I^{1 / 2}\left[\mathrm{~W} / \mathrm{cm}^{2}\right] \simeq 1.4$ with full energy and optimum compression beam $(\simeq 40 \mathrm{fs}$ $40 \mathrm{TW}$ ). Hydrogen gas was introduced into the capillary waveguide using two gas slots (see Fig. 1 upper inset). A discharge was struck between two electrodes located at each end of the waveguide, using a high voltage pulsed power supply that utilized a $2.7 \mathrm{nF}$ capacitor charged to 20 $\mathrm{kV}$. Measurements $[7,8]$ and modeling [9] showed that a fully ionized, approximately parabolic channel is formed. This is also confirmed by the absence of significant ionization induced blueshifting of the laser spectrum when proper guiding conditions are achieved. Previous experiments [10] demonstrated channeling of non-relativistically intense laser pulses with $I \lesssim 10^{17} \mathrm{~W} / \mathrm{cm}^{2}$ in $30-50 \mathrm{~mm}$ long capillaries, which did not generate electron beams.

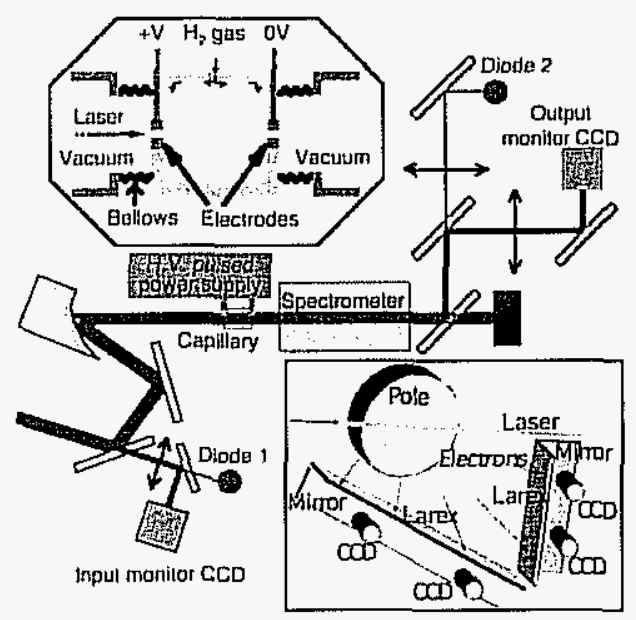

Figure 1: Schematic diagram of the capillary dischargeguided laser wakefield accelerator and the diagnostics. The detailed detailed discription for a capillary discharge unit is in upper inset, and the electron spectrometer in lower inset. See manuscript for more information. 
The laser intensity and profile were monitored both before and after an interaction to evaluate the guiding efficiency and guided beam quality. Following the capillary unit was a round dipole magnet with a maximum magnetic field of 1.2 T, which separated an electron beam from the laser beam [6] (see Fig. 1 lower inset). The magnet deflected the electrons vertically downward onto two phosphor screens mounted on the exit flanges of the vacuum chamber. Four synchronously triggered 12-bit chargecoupled device (CCD) cameras imaged a $75 \mathrm{~cm}$ long (bottom) and a $45 \mathrm{~cm}$ long (forward) phosphor screen, allowing simultaneous single shot measurement of electrons from $0.03 \mathrm{GeV}$ to $0.15 \mathrm{GeV}$ (bottom) and $0.17 \mathrm{GeV}$ to $1.1 \mathrm{GeV}$ (forward) [11].

\section{RESULTS AND ANALYSIS}

The relativistic electron beam generation via CDGLWFA was experimentally studied by making use of 33 $\mathrm{mm}$ long capillaries with diameters of 190, 225, and $310 \mu \mathrm{m}[5,6]$. Guiding was optimized by adjusting the initial gas density and the delay between onset of the discharge current and arrival of the laser pulse (discharge-laser timing $t_{d l}$ ). The measured discharge current was reproducible with shot-to-shot fluctuations of a few $\%$, and had periodic ripples on its profile. The laser beam transmission was found to be above $90 \%$ for low power operation $(<5 \mathrm{TW})$, and became lower when the intensity was sufficiently high for wakefield generation. Note that the jitter between the arrival time of the laser and the discharge current is not an intrinsic problem for the waveguide, because the $\sim 100 \mathrm{~ns}$ timing window is far larger than the jitter in the system which is a few ns. Without a preformed plasma channel (laser injected ahead of discharge), the transmission efficiency went down below $5 \%$ and bulk damage was sustained to the capillary channel walls, indicating that self-ionization and relativistic self-focusing could not be relied on for guiding, as expected from short pulse propagation theory [12]. The parameters of the generated electron beams were found to be sensitive and exhibited a complicated interdependence on input laser and plasma parameters. By taking advantage of the high repetition rate experimental system, data sets containing several thousands shots were taken and sorted into sets with similar parameters, allowing a statistical evaluation of the overall performance.

The reproducible generation of a $0.5 \mathrm{GeV}$ beam with $225 \mu \mathrm{m}$ diameter capillary was reported previously $[5,6]$. In the vicinity of this regime, the results were analyzed against the discharge-laser timing $t_{d l}$ and laser intensity. Shots within the range of $0.65<a_{0}<0.8$ and $140<$ $t_{d t}<180 \mathrm{~ns}$ were taken into account for a plasma density of $3.5 \times 10^{18} / \mathrm{cm}^{3}$. Note that here the density means the estimated axial electron density from the measured Hydrogen gas pressure inside of the capillary using the scaling law from Gonsalves et al [8].

Shown in Fig. 2 are the beam peak energy and total charge versus the the discharge-laser timing. One can see that the beam energy was high and beam charge was low at $\simeq 150 \mathrm{~ns}$. This is where the mono-energetic beams were observed. When the timing was not in this regime, the resultant electron beams contained higher charge and lower peak energy with broader energy spread. The normalized vector potential dependence of the beam peak energy and total charge is shown in Fig. 3. The laser pulse duration was kept at $70-80 \mathrm{fs}$, and the difference of the intensity comes from varying the laser energy, which was $0.9 \mathrm{~J}$ with $\pm 10 \%$ fluctuation. The laser beam intensity dependence shows that $\simeq 0.5 \mathrm{GeV}$ electron beams containing 10 's of $\mathrm{pC}$ were generated with laser power below $a_{0} \simeq 0.78$. Above that laser intensity, electron beam energy starts to decrease while total charge of the beam increases, consistent with the higher amount of charge resulting in earlier beam loading [13].

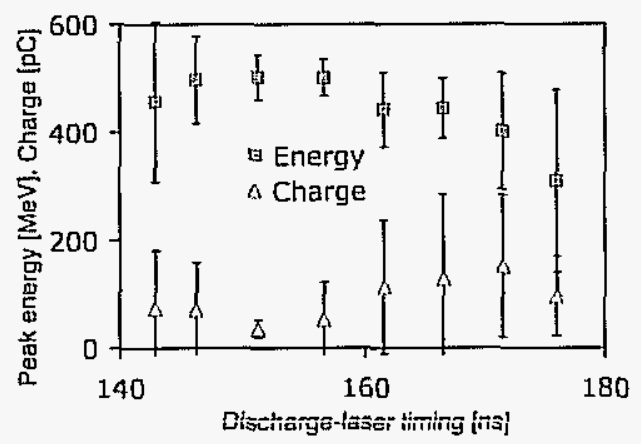

Figure 2: Peak electron beam energy (square) and total charge (triangle) versus the laser-discharge timing.

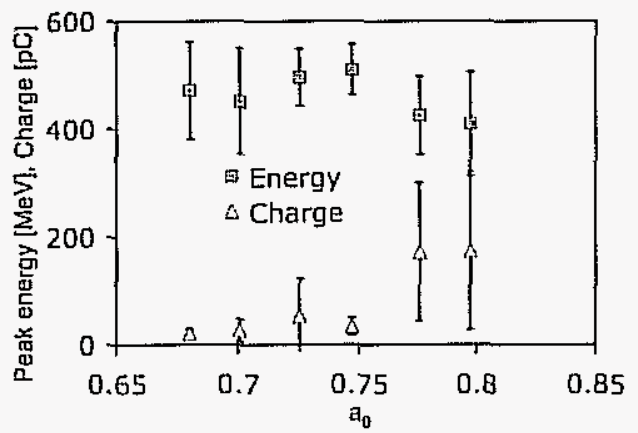

Figure 3: Peak electron beam energy (square) and total charge (triangle) versus the normalized vector potential.

The beam divergence and the pointing stability versus (he discharge-laser timing are shown in Fig. 4. The beam divergence of each shot was obtained from the root mean square (rms) width of the energy-integrated profile (horizontal profile). The peak value of the horizontal profile for each shot was used to evaluate a beam propagation angle. Then the rms fluctuation of the propagation angles was statistically computed to address the beam pointing stability, which was used to define the error bar of the absolute beam 
energy [6]. Beams exhibited smaller divergences where mono-energetic beams were found. While a dependence of the beam divergence on the laser-discharge timing was seen, it was found to be independent on laser intensity over the range $0.65<a_{0}<0.8$ as shown in Fig. 5. Both the beam divergence and pointing stability are weakly correlated with $a_{0}$, which implies a weak correlation with charge from Fig. 3. total charge. This suggests that in this regime, the beam divergence depends more on the plasma channel condition (controlled via discharge-laser timing) than on the input laser intensity.

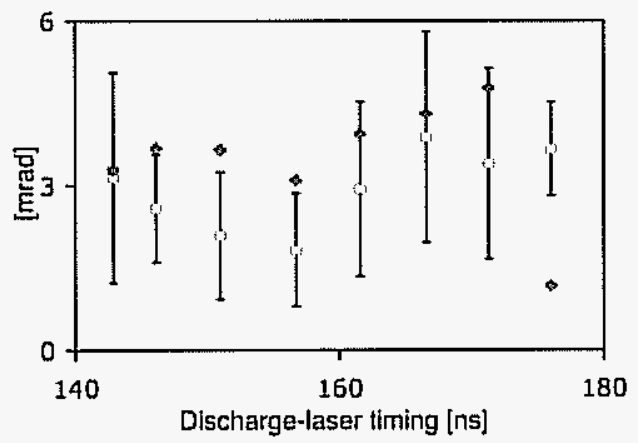

Figure 4: Electron beam pointing stability (diamond) and rms divergence (circle) versus the laser-discharge timing.

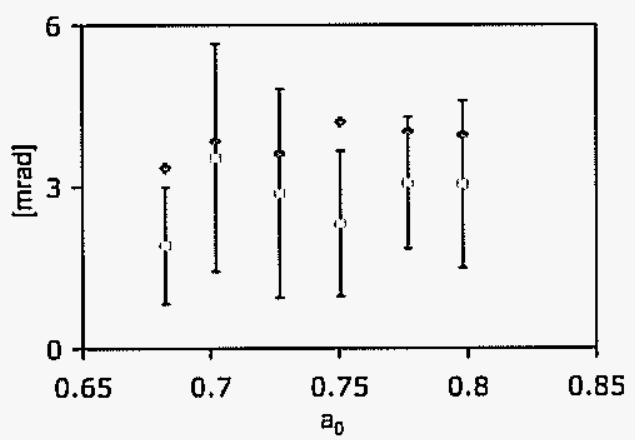

Figure 5: Electron beam pointing stability (diamond) and rms divergence (circle) versus the normalized vector potential.

\section{SUMMARY}

The operation of a CDG-LWFA with a capillary of diameter $225 \mu \mathrm{m}$ and length $33 \mathrm{~mm}$ was analyzed. The discharge-laser timing was found to be critical for the mono-energetic beam generation, pointing stability and beam divergence. The input laser intensity seems to affect the amount of injected electrons into a wakefield. Increasing input intensity leads to broadband and high charge beam, which may be explained by earlier beam loading. The dependence of the beam divergence on the dischargelaesr timing suggests that the spatial quality of electron beams can be controlled with plasma channel parameters.
Although modeling and further study with particle-in-cell simulations are required to understand the detailed physics of injection and acceleration of a CDG-LWFA, the analysis presented in the paper provides information for future optimization.

\section{REFERENCES}

[1] T. Tajima and J. M. Dawson, Phys. Rev. Lett. 43, 267 (1979); E. Esarey, P. Sprangle, J. Krall, and A. Ting, IEEE Trans. Plasma Sci. 24, 252 (1996).

[2] For example, S. P. D. Mangles, C. Murphy, Z. Najmudin, A. Thomas, J. Collier, A. Dangor, E. Divali, P. Foster, J. Gallacher, C. Hooker, D. Jaroszynski, A. Langley, W. Mori, P. Norreys, F. Tsung, R. Viskup, B. Walton, and K. Krushelnick, Nature 431, 535 (2004); C. G. R. Geddes, C. Tóth, J. van Tilborg, E. Esarey, C. B. Schroeder, D. Brthwiler, C. Nieter, J. Cary, and W. P. Leemans, Nature 431, 538 (2004); J. Faure, Y. Glinec, A. Pukhov, S. Kiselev, S. Gordienko, E. Lefebvre, J.-P. Rousseau, F. Burgy, and V. Malka, Nature 431, 541 (2004); T. Hosokai, K. Kinoshita, A. Zhidkov, A. Maekawa, A. Yamazaki, and M. Uesaka, Phys. Rev. Lett. 97, 075004 (2006); I. Blumenfeld, C. E. Clayton, F.J. Decker, M. J. Hogan, C. Huang, R. Ischebeck, R. Iverson, C. Joshi, T. Katsouleas, N. Kirby, W. Lu, K. A. Marsh, W. B. Mori, P. Muggli, E. Oz, R. H. Siemann, D. Walz, and M. Zhou, Nature 445, 741 (2007).

[3] J. van Tilborg, C. B. Schroeder, C. V. Filip, C. Tóth, C. G. R. Geddes, G. Fubiani, R. Huber, R. A. Kaindl, E. Esarey, and W. P. Leemans, Phys. Rev. Lett. 96, 014801 (2006).

[4] Y. Muroya, M. Lin, T. Watanabe, G. Wu, T. Kobayashi, K. Yoshii, T. Ueda, M. Uesaka, and Y. Katsumura, Nuclear Instruments and Methods in Physics Research Section A 489, 554 (2002).

[5] W. P. Leemans, B. Nagler, A. J. Gonsalves, C. Tóth, K. Nakamura, C. G. R. Geddes, E. Esarey, C. B. Schroeder, and S. M. Hooker, Nature Physics 2, 696 (2006).

[6] K. Nakamura, B. Nagler, C. Tóth, C. G. R. Geddes, C. B. Schroeder, E. Esarey, W. P. Leemans, A. J. Gonsalves, and S. M. Hooker, Phys. Plasmas 14, 056708 (2007).

[7] D. J. Spence and S. M. Hooker, Phys. Rev. E 63, 015401(R) (2001).

[8] A. J. Gonsalves, T. P. Rowlands-Rees, B. H. P. Broks, J. J. A. M. van der Mullen, and S. M. Hooker, Phys. Rev. Lett. 98, 025002 (2007).

[9] N. A. Bobrova, A. A. Esaulov, J. Sakai, P. V. Sasorov, D. J. Spence, A. Butler, S. M. Hooker, and S. V. Bulanov, Phys. Rev. E 65, 016407 (2002); B. H. P. Broks, K. Garloff, and J. J. A. M. van der Mullen, Phys. Rev. E 71, 016401 (2005).

[10] A. Butler, D. J. Spence, and S. M. Hooker, Phys. Rev. Lett. 89,185003 (2002).

[11] K. Nakamura, et al., in preparation.

[12] P. Sprangle, E. Esarey, and A. Ting, Phys. Rev. Lett. 64, 2011 (1990).

[13] F. S. Tsung, R. Narang, W. B. Mori, C. Joshi, R. A. Fonseca, and L. O. Silva, Phys. Rev. Lett. 93, 185002 (2004); C. G. R. Geddes, C. Tóth, J. van Tilborg, E. Esarey, C. B. Schroeder, D. Bruhwiler, C. Nieter, J. Cary, and W. P. Leemans, Phys. Plasmas 12, 056709 (2005). 